\title{
Suppression of bladder cancer cell tumorigenicity in an athymic mouse model by adenoviral vector-mediated transfer of LRIG1
}

\author{
FAN LI ${ }^{1}$, ZHANG-QUN YE ${ }^{1}$, DONG-SHENG GUO ${ }^{2}$ and WEI-MIN YANG ${ }^{1}$ \\ Departments of ${ }^{1}$ Urology and ${ }^{2}$ Neurosurgery, Tongji Hospital, Tongji Medical College, \\ Huazhong University of Science and Technology, Wuhan, Hubei, P.R. China
}

Received March 6, 2011; Accepted April 8, 2011

DOI: 10.3892/or.2011.1304

\begin{abstract}
Previous in vitro studies demonstrated that leucinerich repeats and immunoglobulin-like domains 1 (LRIG1), a negative regulator of EGFR, is a novel agent for suppressing bladder cancer. However, the role that LRIG1 plays in bladder cancer growth in vivo has not been elucidated in animal or clinical studies. Thus, to evaluate the suppressive effects of LRIG1 on human invasive bladder cancer in vivo, we transferred LRIG1 into athymic mice bearing T24 invasive bladder cancer xenografts mediated by an adenoviral vector. Correlations between LRIG1 expression and tumorigenicity, EGFR expression, xenograft proliferation and angiogenesis were assessed, respectively. The results indicated that tumor volume growth was retarded via Ad-LRIG1 intratumoral injection. In addition LRIG1 upregulation was well correlated with a reduction in EGFR expression and the proliferation rate of xenografts. Furthermore, microvessel densities were reduced and correlated with Ad-LRIG1 administration. No significant macroscopic and microscopic pathological abnormality was observed in the liver, kidney and lungs of Ad-LRIG1-administered mice. This new insight provides evidence that downregulation of EGFR expression by LRIG1 may comprise a potential novel and safe therapeutic approach for improving the prognosis of invasive bladder cancer.
\end{abstract}

Correspondence to: Dr Wei-Min Yang, Department of Urology, Tongji Hospital, Tongji Medical College, Huazhong University of Science and Technology, Wuhan, Hubei 430030, P.R. China E-mail: wmyang@tjh.tjmu.edu.cn

Abbreviations: DMEM, Dulbecco's modified Eagle's medium; EGFR, epidermal growth factor receptor; FBS, fetal bovine serum; FITC, fluorescein isothiocyanate; GFP, green fluorescence protein; H\&E, hematoxylin and eosin; LRIG1, leucine-rich repeats and immunoglobulin-like domains 1; MVD, microvessel density; PCNA, proliferation cell nuclear antigen; SPF, specific-pathogen free

Key words: bladder cancer, epidermal growth factor receptor, leucinerich repeats and immunoglobulin-like domains 1, target therapy

\section{Introduction}

Bladder cancer, mostly urothelial or transitional carcinoma, is a common malignant lesion jeopardizing the function of the urinary system. Seventy percent of bladder cancer patients are diagnosed with non-muscle-invasive cancer, and 20-30\% of patients present with muscle-invasive cancer (1). Non-muscleinvasive cancer is managed by transurethral surgery, intravesical chemotherapy or immunotherapy and is associated with an optimistic prognosis, yet recurrence is commonly noted and is associated with progression to muscle-invasive cancer (2). Patients presenting with muscle-invasive cancer or progressing to this stage have a poor survival rate, despite receiving conventional therapies (2). Thus, effective agents must be developed to control the recurrence rate and enhance the survival rate of invasive bladder cancer.

Epidermal growth factor receptor (EGFR) is a transmembrane protein tyrosine kinase and overexpressed or activated in a variety of malignant lesions, including bladder cancer (3). Overexpressed or activated EGFR signaling is the initial step of a cascade of events leading to tumor cell proliferation, invasion, migration and evasion of apoptosis $(4,5)$. Preclinical and clinical studies have shown that a target blocking EGFR binding sites and/or EGFR-specific tyrosine kinase attenuated tumor cell proliferation and invasion $(6,7)$. Therefore, EGFR is a potential target for molecular therapy for invasive bladder cancer.

Leucine-rich repeats and immunoglobulin-like domains 1 (LRIG1) is a transmembrane leucine-rich repeat and immunoglobulin (Ig)-like domain-containing protein, whose transcript is located at chromosome 3 p14.3, a region frequently deleted in various types of human cancers (8). Studies have demonstrated that LRIG1 is downregulated in skin, kidney, bladder, cervix and lung carcinomas (7-9). Further studies revealed that LRIG1 is one of the natural ligands of EGFR and acts as a negative regulator of the ErbB family of receptor tyrosine kinases (10). Upregulation of the LRIG1 transcript and protein levels was found to restrict EGFR signaling in several cancer cell lines, and is associated with LRIG1-EGFR complex formation, ubiquitylation and degradation (10). In our previous study, LRIG1 was found to be absent or downexpressed in human malignant bladder tissues, with an upregulation of EGFR expression and activity, when compared to human normal bladder tissues. Upregulation of LRIG1 expression by 
plasmid transfection in the superficial bladder cancer BIU-87 cell line resulted in inhibition of cell proliferation, promotion of cell apoptosis, cell cycle arrest and attenuation of cell metastatic and invasive abilities in vitro, indicating that LRIG1 is an attractive tumor-suppressor gene (TSG) in bladder cancer (7). The in vitro study described above was performed to ascertain whether LRIG1 is a novel agent in suppressing bladder cancer in patients. However, the role of LRIG1 in suppressing bladder cancer growth in vivo has not previously been investigated in animal or clinical studies.

Based on the evidence described above, a preclinical study to identify LRIG1 as a tumor-suppressing agent which inhibits invasive bladder cancer-induced tumorigenicity and angiogenesis in vivo was carried out. Here, athymic mice bearing T24 invasive bladder cancer cells were administered LRIG1 mediated by adenoviral vector. Characterizations of xenograft growth, in regards to tumor volume, proliferation and angiogenesis were assessed.

\section{Materials and methods}

Cell line and cell culture. The human bladder cancer cell line T24 was kindly provided by Dr Zhulin Liang. Cells were maintained in Dulbecco's modified Eagle's medium (DMEM), high glucose, supplemented with $10 \%$ (v/v) fetal bovine serum (FBS) (Gibco BRL, Gaithersburg, MD, USA) at $37^{\circ} \mathrm{C}$ in a humidified atmosphere of $5 \% \mathrm{CO}_{2}$. The medium was replaced every 2-3 days. Cells were passaged every 5-6 days.

Construction of adenoviral vector encoding human LRIG1. The plasmid pLRIG1-GFP containing full-length human lrig1 cDNA was kindly provided by Professor Håkan Hedman and Dr Jonas Nilsson (Umeå University Hospital, Sweden). Human lrig1 cDNA was amplified from plasmid pLRIG1-GFP by PCR. The forward PCR primer was 5'-GTA GAA CGC AGA TCGAATTCA TGG CGC GGC CGG TC-3', and the reverse primer was 5'-CCC TTG CTC ACC ATGAATTCG CTT TTT GGT GCC AAC AGC-3'. Recombinant adenovirus encoding human lrig1 (Ad-LRIG1) was constructed using the shuttle plasmid pDC315-GFP-LRIG1 (Genechem Gene Technology Company Ltd., Shanghai, China) and the helper plasmid pBHGlox (ס) E1, 3 Cre, was then encoated using the AdMax Kit D (Microbix Biosystems Inc., Canada). Ad-GFP, a recombinant adenovirus carrying the green fluorescent protein (GFP) gene, was produced similarly as the control vector.

Establishment of a human bladder cancer model in athymic mice. Four-week-old male athymic mice (Balb/c-nu/nu), weighing 18-20 g, were purchased from Shanghai Laboratory Animal Center, Chinese Academy of Sciences (SLAC, Shanghai, China), and were bred in a sterilized, specific pathogen-free (SPF) environment at $25-27^{\circ} \mathrm{C}$, with a constant humidity (45-50\%) and dust-free fresh air. T24 cells in the logarithmic growth phase were trypsinized, centrifuged and resuspended in DMEM. After ensuring that the cell survival rate was $>90 \%$ by trypan blue, the cell concentration was adjusted to $5 \times 10^{7} / \mathrm{ml}$ with DMEM, and $200 \mu \mathrm{l}$ of the cell suspension was subcutaneously injected into the right flanks of the athymic mice using a 6-gauge needle. After tumors reached maximum diameters of $\sim 10 \mathrm{~mm}$, mice were anesthetized by inhalation of $5 \%$ ethoxyethane and sacrificed. The xenografts were resected, fixed in $4 \%$ paraformaldehyde, embedded in paraffin wax, sectioned serially $(4-\mu \mathrm{m})$, and stained with hematoxylin and eosin (H\&E). The properties of the tumor xenografts were confirmed under light microscopy. The animal experiments were approved by the Institutional Animal Care and Use Committee and in compliance with all regulatory institutional guidelines for animal welfare.

Adenoviral vector-mediated LRIGI administration in vivo. The T24 bladder cancer cell xenograft model was established as described above and carried out until the tumor maximum diameters reached $\sim 5 \mathrm{~mm}$. Then mice were randomly divided into 3 groups ( $n=6)$ : DMEM group (blank control), Ad-GFP group (negative control), and Ad-LRIG1 group (treatment group). Subsequently, $100 \mu 1$ of DMEM alone, or a suspension of adenovirus $\left(1 \times 10^{9} \mathrm{pfu}\right)$ in $100 \mu \mathrm{l}$ of DMEM was administered by intratumoral injection into the tumor-bearing mice in each group twice a week for a total of 6 injections per mouse $(11,12)$. Long diameters (a) and short diameters (b) of the tumors were measured every 3-4 days. Volumes (V) of the tumors were calculated according to the formula: $\mathrm{V}=0.5 \mathrm{x} \mathrm{a} \mathrm{x} \mathrm{b}^{2}$, and a tumor growth curve was constructed. Inhibition of tumor growth was determined using the formula: Tumor inhibition rate $(\%)=($ volume of tumors in the control group - volume of tumors in the treatment group)/volume of tumors in the control group x $100 \%$. Three weeks after the final injections, all mice were anesthetized by ethoxyethane and sacrificed. The tumors were resected, fixed in methanol for GFP fluorescent quenching and in $4 \%$ paraformaldehyde, and lungs, livers and kidneys were resected, fixed in $4 \%$ paraformaldehyde for at least $24 \mathrm{~h}$, embedded in paraffin wax, and serially sectioned $(4-\mu \mathrm{m})$.

Immunofluorence detection and scoring of LRIGI expression. Immunofluorescence staining was performed according to a standard protocol (13). Slides were incubated with a polyclonal goat anti-LRIG1 antibody 1:200 (sc-50076; Santa Cruz Biotechnology, USA), followed by a donkey anti-goat IgG-FITC 1:100 (sc-2024; Santa Cruz Biotechnology). Hoechst 33258 was used to identify cell nuclear expression. A four-grade semi-quantitative score was used to assess LRIG1 expression: 0 , absence of expression; 1 , expression in $1-19 \%$ of cancer cells; 2, 20-49\%; and 3, $\geq 50 \%$ cells (14).

Immunohistochemical detection and scoring of EGFR, PCNA and Ki-67 expression. Immunohistochemical staining was performed according to a standard protocol (15). Slides were probed with a polyclonal rabbit anti-EGFR antibody, 1:250 (sc-03; Santa Cruz Biotechnology), a monoclonal mouse antiPCNA antibody, 1:4000 (PC-10; Cell Signaling Technology Co., USA) and monoclonal mouse anti-Ki-67, 1:300 (sc-23900; Santa Cruz Biotechnology), and followed by a biotinylated polyclonal goat anti-rabbit or goat anti-mouse antibody.

EGFR staining was defined as the immunostaining of tumor cell membranes above the background level and scored as: $1+$, weak expression; $2+$, moderate expression; and $3+$, strong expression, and the absence of membrane or cytoplasmic staining was considered negative. The percentage of stained 

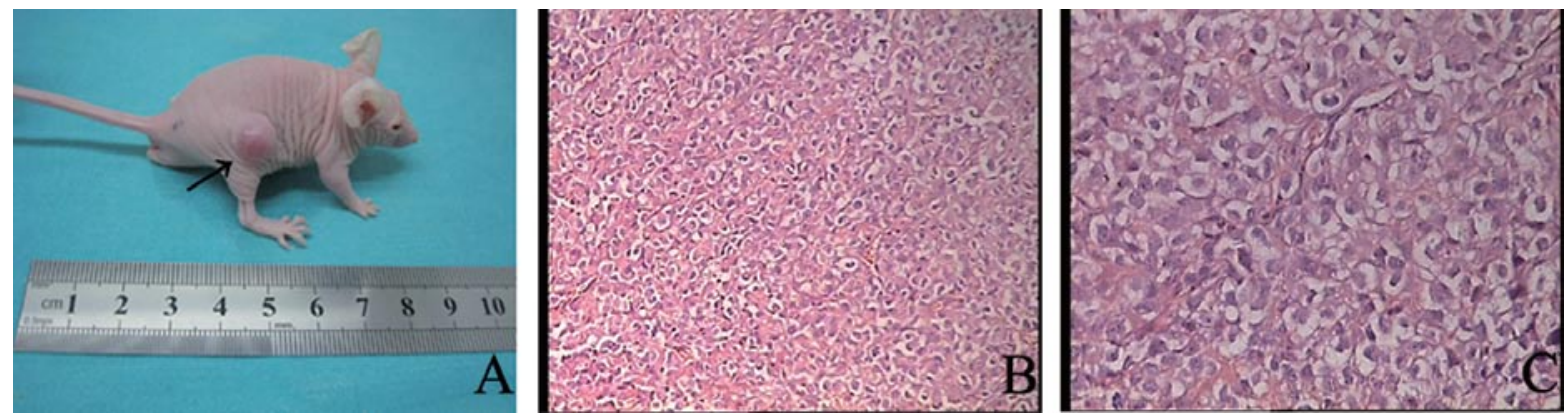

Figure 1. T24 human bladder cancer cell xenograft model established in athymic mice. (A) The T24 bladder cancer cells formed a nodous shape in a subcutaneous xenograft (arrow), in the right flank of an athymic mouse after cell inoculation. Tumors were diagnosed using malignant lesions by slide staining, under light microscopy (H\&E). Photomicrographs were captured at (B) x100 and (C) x200.

cells was assessed as: 0 or $<1 \%$, negative; $1+, 1-10 \% ; 2+$, $10-50 \%$; and $3+,>50 \%(16,17)$. The two scores were combined to represent the final expression assessment.

Proliferating tumor cells were detected by proliferating cell nuclear antigen (PCNA) and Ki-67 staining. The evaluation of PCNA and Ki-67 expression was based on the proportion of positively stained cells, in which the cell nuclei were stained dark brown, among a total of 1000 counted cells. The percentage of labeled nuclei was then calculated and defined as the labeling index (LI \%) (18).

Evaluation of the tumor microvessel density (MVD). The microvessel density (MVD) was evaluated by counting factor VIII (FVIII)-positive stained vessels. Paraffin-embedded sections of tumor xenografts were probed with a polyclonal rabbit anti-FVIII antibody (bs-0332R; Bioss Co. Beijing China), followed by a biotinylated polyclonal goat anti-rabbit antibody. The MVD was determined by light microscopy according to the procedure of Weidner et al (19). For microvessel counting, positive staining for FVIII in the three most highly vascularized areas, known as hot spots, were counted in $\times 200$ fields, and clusters of stained endothelial cells distinct from adjacent microvessels, tumor cells, or other stromal cells were counted as one microvessel (19). The density of microvessels was expressed as the total number of micro-vessels per slide.

Evaluation of the safety of the adenoviral vector administration. During the administration course, safety of the adenoviral vector administration was mainly assessed by indices such as behavior, food intake, fluid intake and weight change of the mice $(20,21)$. Lung, liver and kidney tissues were resected, fixed, embedded in paraffin, and serially sectioned (4- $\mu \mathrm{m})$. Slides were then stained with H\&E and observed under light microscopy.

Statistical analysis. Statistical analyses were performed using SPSS 13.0 Statistical Software (SPSS Inc., Chicago, IL). ANOVA and the Kruskal-Wallis test were used to compare data among multiple groups. The S-N-K method was used for paired comparison. Spearman rank correlation was used to assess the relationship between two variables. Significance was defined as $\mathrm{p}<0.05$.

\section{Results}

Human bladder cancer model established in athymic mice. The T2 4 cells formed oval or nodous shapes in smooth surface subcutaneous xenografts, $\sim 10 \mathrm{~mm}$, in the right flanks of athymic mice after cell inoculation (Fig. 1A). H\&E staining confirmed the tumors as bladder transitional cell carcinoma. Tumor cells were noted with obvious heteromorphism microscopically, and the cell nuclei were large and irregular with obvious nucleoli (Fig. 1B and C).

Ad-LRIG1 inhibits xenograft growth. Subcutaneous inoculation of T24 cells resulted in tumor formation in all three groups of athymic mice. After the maximum diameters of the tumors reached $5 \mathrm{~mm}, \sim 7-10$ days after inoculation, mice were administered DMEM (control), Ad-GFP and Ad-LRIG1, respectively.

Tumor growth curves showed that the average size of tumors in the Ad-LRIG1 group was significantly smaller compared to the other two groups, while no significant difference was observed between the Ad-GFP and control group $\left(1238.46 \pm 546.52 \mathrm{~mm}^{3}\right.$ in Ad-LRIG1 vs. $2201.39 \pm 547.09 \mathrm{~mm}^{3}$ in control group and $2434.28 \pm 398.47 \mathrm{~mm}^{3}$ in the Ad-GFP group; p<0.05) (Fig. 2). The tumor growth inhibition rates in the Ad-LRIG1 group were $43.74 \%$ and $49.12 \%$, compared to the control and Ad-GFP groups, respectively. These results confirmed that Ad-LRIG1 administration via intratumoral injection effectively inhibits tumor growth.

No significant difference in body weight among the three groups before or after administration were noted (data not shown). After the slides were H\&E stained, no metastases in the organs of the thoracic (lungs) or abdominal (liver and kidneys) cavity were found. No animals died during the administration and observation period.

According to the results of the slide staining, upon the upregulation of LRIG1, the staining scores and areas of EGFR were attenuated compared to the control groups $(p<0.05)$ (Figs. 3 and 4). A significant negative correlation was found between LRIG1 and EGFR expression levels ( $\mathrm{r}=-0.51, \mathrm{p}=0.03$ ).

As a biomarker of proliferation and malignancy in tumors, PCNA and Ki-67 immunohistochemical staining was used to assess the efficiency of Ad-LRIG1. PCNA and Ki-67 were downregulated significantly in the Ad-LRIG1 group compared 


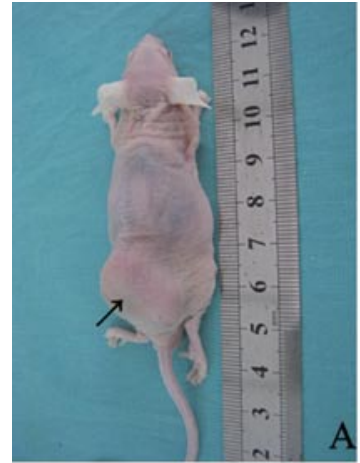

Control

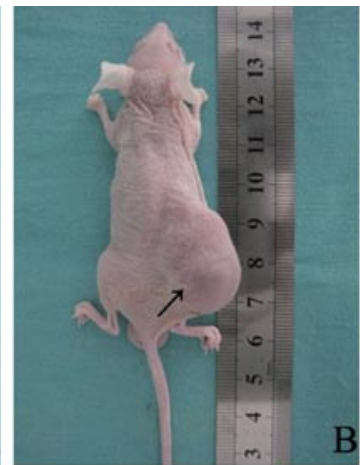

Ad-GFP

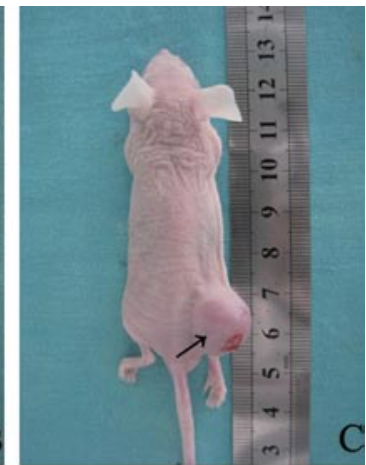

Ad-LRIG1

$\mathbf{D}$

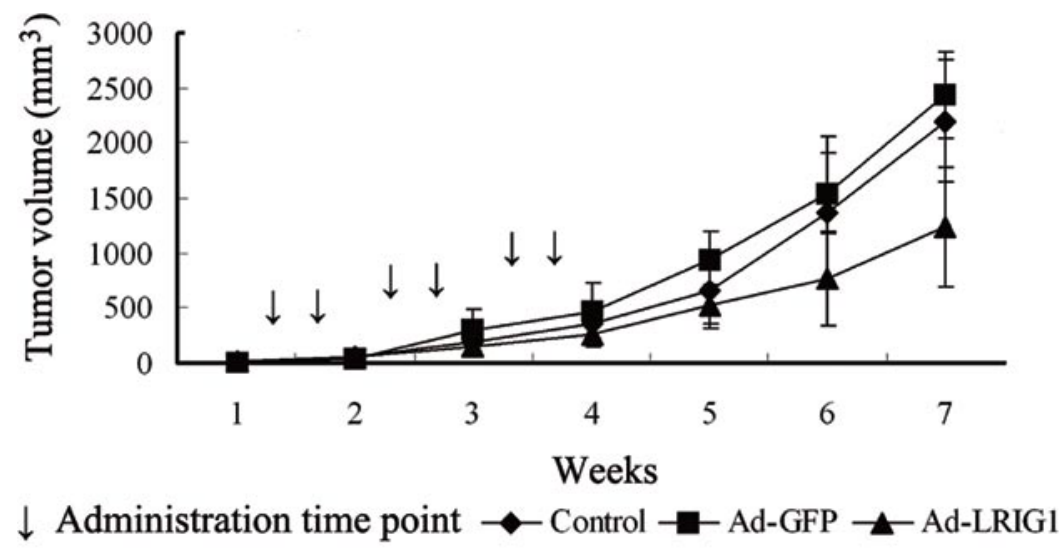

Figure 2. Tumor volume growth was retarded by Ad-LRIG1 intratumoral injection. Athymic mice were divided into 3 groups and treated with (A) DMEM as control, (B) Ad-GFP and (C) Ad-LRIG1, respectively. Three weeks after the final administration, xenograft volume growth (arrow) was obviously suppressed by 6 intratumoral injections of Ad-LRIG1. (D) Tumor growth curves indicated that the average size of the tumors in the Ad-LRIG1 group was significantly smaller than that in the other groups at the conclusion of the study $(\mathrm{p}<0.05)$.

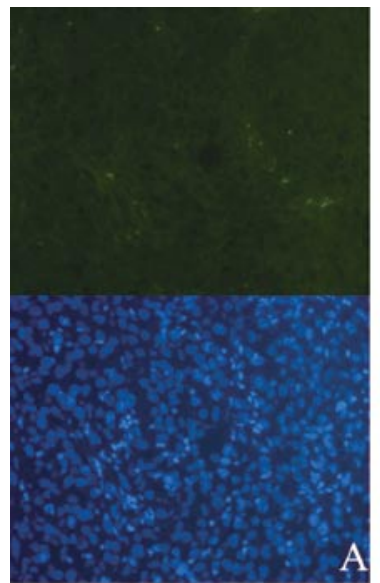

Control

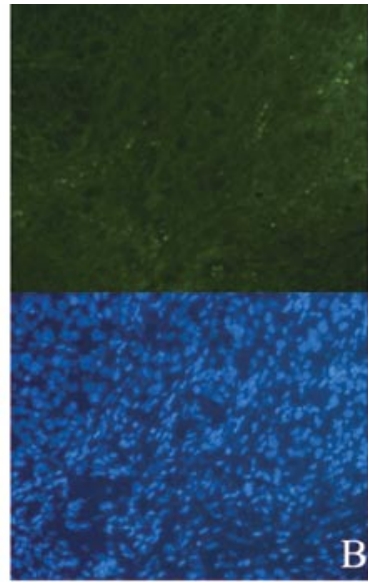

Ad-GFP

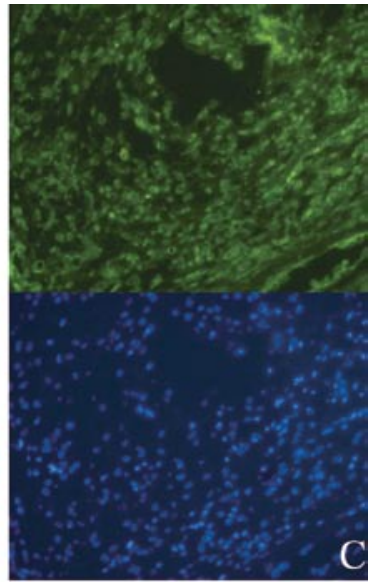

Ad-LRIG1

Figure 3. LRIG1 was overexpressed in the xenografts treated by the adenoviral vector. Athymic mice were intratumorally injected with (A) DMEM as control, (B) Ad-GFP and (C) Ad-LRIG1, respectively. LRIG1 was probed using an immunofluorescence staining method in paraffin-embedded tumor tissues. LRIG1 expression was indicated by green fluorescence staining using FITC, and cellular nuclear expression was indicated by blue fluorescence staining using Hoechst 33258. LRIG1 was successfully transducted and overexpressed in the xenograft tissues by the adenoviral vector.

to the control and Ad-GFP groups (Figs. 5A-C and 6A-C) (PCNA LI value: $32.48 \pm 15.35 \%$ in the Ad-LRIG1 group vs. $64.45 \pm 26.46 \%$ in the control group and $60.89 \pm 19.19 \%$ in the Ad-GFP group; p<0.05) (Fig. 5D); (Ki-67 LI value: $16.04 \pm 8.72 \%$ in the Ad-LRIG1 group vs. $35.42 \pm 14.17 \%$ in the control group and $37.59 \pm 17.57 \%$ in the Ad-GFP group; $\mathrm{p}<0.05$ ) (Fig. 6D). Significant negative correlations were found between LRIG1 and PCNA $(\mathrm{r}=-0.589, \mathrm{p}=0.01)$ and $\mathrm{Ki}-67(\mathrm{r}=-0.548, \mathrm{p}=0.019)$. The immunohistochemical staining results of PCNA and Ki-67 revealed that LRIG1 may reduce bladder cancer growth in vivo by inhibiting cancer cell proliferation. 


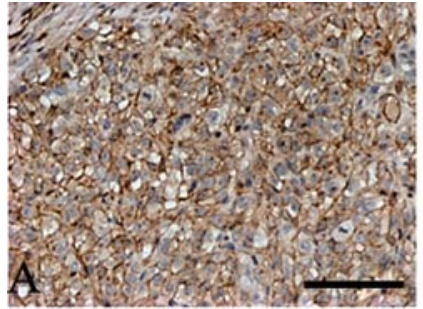

Control

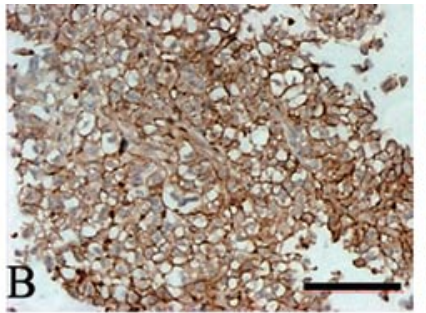

Ad-GFP

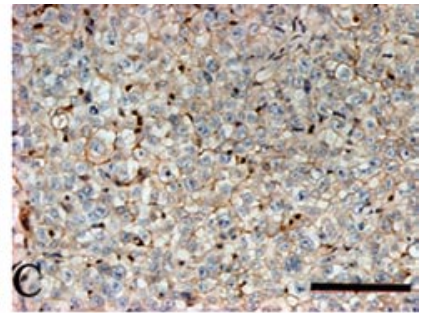

Ad-LRIG1

Figure 4. EGFR expression levels were downregulated after Ad-LRIG1 administration, which correlated with LRIG1 upregulation. Athymic mice were administered (A) DMEM as control, (B) Ad-GFP and (C) Ad-LRIG1, respectively, and EGFR expression levels were probed using an immunohistochemical staining method. Compared to control and Ad-GFP groups, intensities and areas of EGFR expression were attenuated in the Ad-LRIG1-treated xenograft tissues. (Bar, $100 \mu \mathrm{m}$ ).

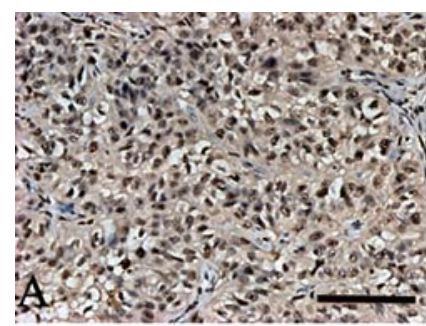

Control

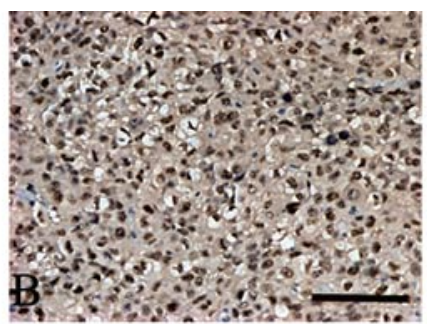

Ad-GFP

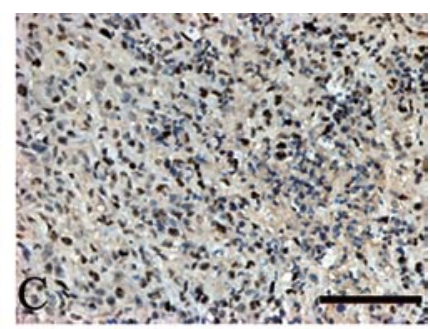

Ad-LRIG1

D

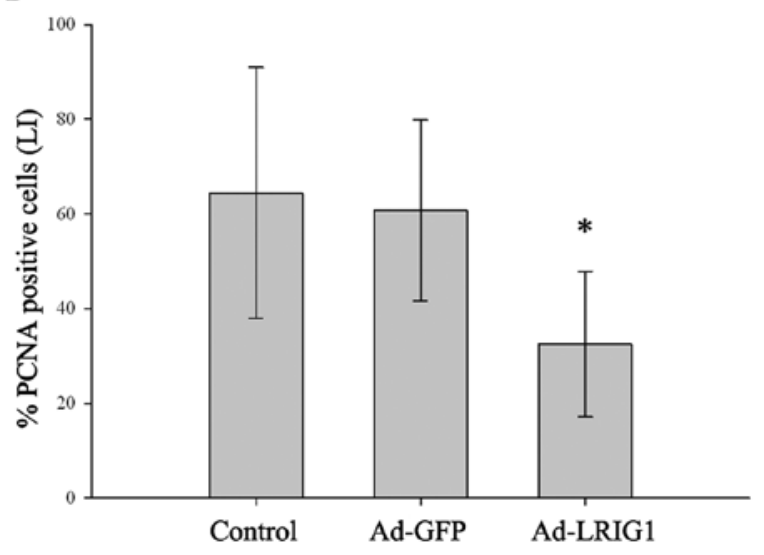

Ad-LRIG1 inhibits angiogenesis in xenografts. Angiogenesis is one of the most critical determinant factors for tumor survival in vivo. Tumor angiogenesis in the bladder cancer athymic mouse model was dominantly reduced by LRIG1 administration (Fig. 7A-C). Tumor MVD values were 19.83 \pm 8.06 in the Ad-LRIG1 group vs. $37.17 \pm 17.76$ in the control group and $42.33 \pm 15.44$ in the Ad-GFP group ( $<<0.05)$ (Fig. 7D), implying that inhibition of tumor angiogenesis may be another potential pathway involved in restrained tumor growth by LRIG1.

Evaluation of the safety of the administration of the adenoviral vector. Concerning the safety of the administration of the adenoviral vector-mediated LRIG1 delivery via intratumoral injection, we monitored and compared the mental and physical conditions of the mice in the Ad-LRIG1 and Ad-GFP groups to the control group. No discernible abnormality was observed, such as behavior, food and fluid intake, and body weight. No
Figure 5. The proliferation rate was assessed by PCNA and Ki-67 immunohistochemical staining. PCNA expression levels were attenuated after Ad-LRIG1 administration. Representative immunohistochemical results of PCNA staining in the (A) control, (B) Ad-GFP- and (C) Ad-LRIG1-treated groups. Immunohistochemical staining was scored as described in Materials and methods. (Bar, $100 \mu \mathrm{m}$ ). (D) Bar graph showing the percentage of positive PCNA-stained cells (PCNA-labeling index) in the Ad-LRIG1 group was significantly lower than that in the control and Ad-GFP groups $\left({ }^{*} \mathrm{p}<0.05\right)$.

signs of adverse effects were observed, and none of the experimental mice died during the procedure or observation period. No macroscopical pathologic changes were observed in the liver, lung and kidney of all mice. Tissue slides of the lung and kidney from the adenoviral vector-administered mice showed no abnormality. Moreover, similar to other studies, H\&E stained slides of the liver showed mild lymphocyte infiltration around some central veins in liver lobules, which may be associated with the immunogenicity of the adenoviral vector, but not the ribonuclease transduction (Fig. 8) $(21,22)$.

\section{Discussion}

The feasibility and efficacy of the inhibitory effects of LRIG1 on EGFR signaling activity have been studied in vitro experiments using the kidney 293 cell line, NIH3T3 fibroblasts, keratinocytes, glioma cells and bladder cancer cells $(9,14,23)$. However, the role of LRIG1 in cancer regulation is still 


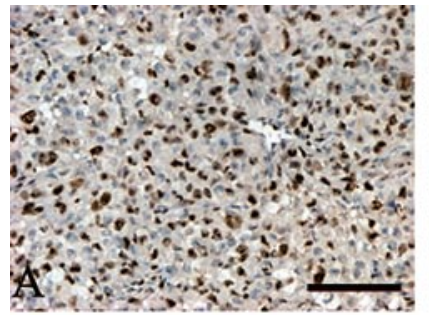

Control

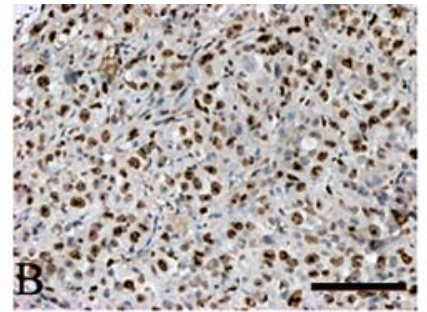

Ad-GFP

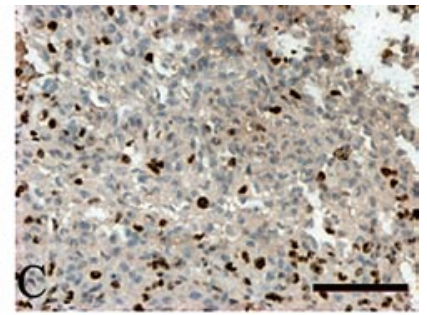

Ad-LRIG1

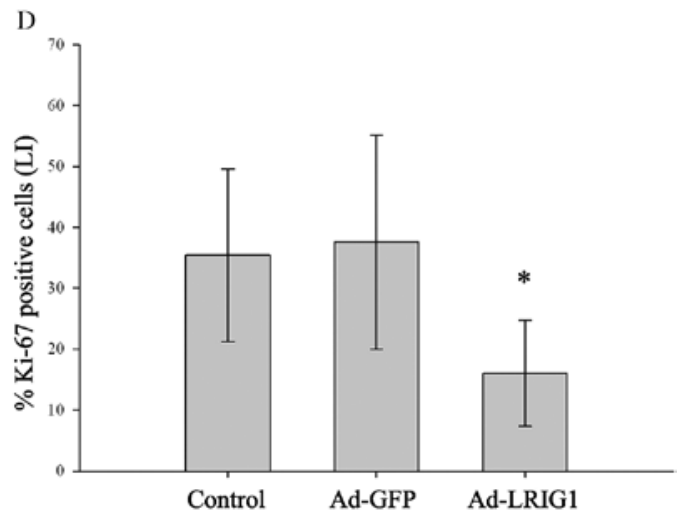

Figure 6 . The proliferation rate was assessed by detecting PCNA and Ki-67 immunohistochemical staining. Ki-67 expression levels were attenuated after Ad-LRIG1 administration. Representative immunohistochemical results of Ki-67 staining in the (A) control, (B) Ad-GFP- and (C) Ad-LRIG1treated groups. Immunohistochemical staining evaluated as described in Materials and methods. (Bar, $100 \mu \mathrm{m}$ ). (D) Bar graph showing that the percentage of positive $\mathrm{Ki}$-67-stained cells (Ki-67-labeling index) in the Ad-LRIG1 group was attenuated compared to the control and Ad-GFP group $(\mathrm{p}<0.05)$.

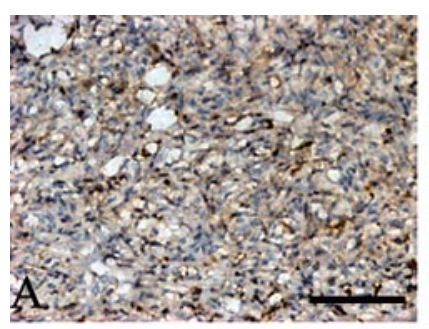

Control

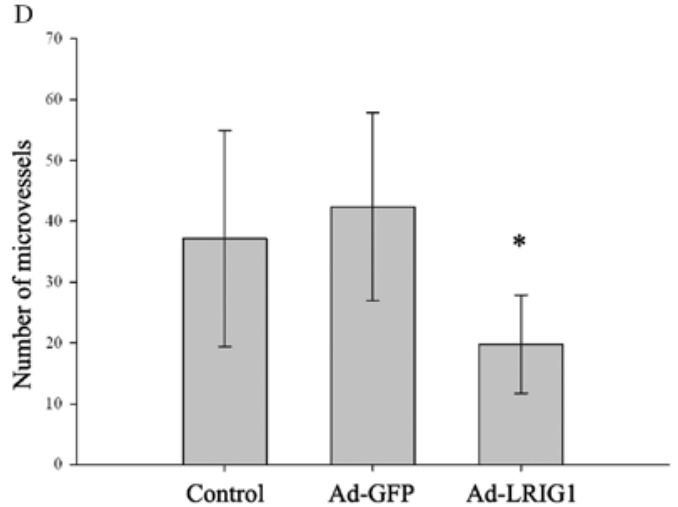

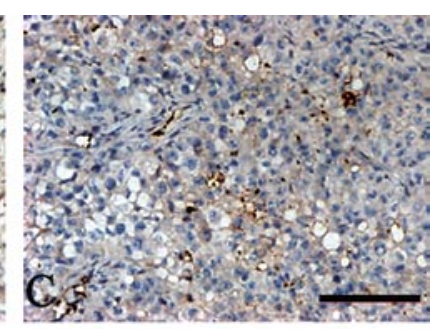

Ad-LRIG1

Figure 7. Reduced angiogenesis in Ad-LRIG1-treated xenografts. The angiogenesis of xenografts was evaluated by immunohistochemical analysis of FVIII, which was predominantly localized in the tumoral endothelial cells. Administration of (C) Ad-LRIG1 showed attenuation of FVIII staining in contrast to the (A) DMEM (control) and (B) Ad-GFP group. (Bar, $100 \mu \mathrm{m}$ ). (D) Bar graph indicating that the microvessel density in the Ad-LRIG1 group was significantly reduced compared to the control and Ad-GFP group $\left({ }^{*} \mathrm{p}<0.05\right)$.
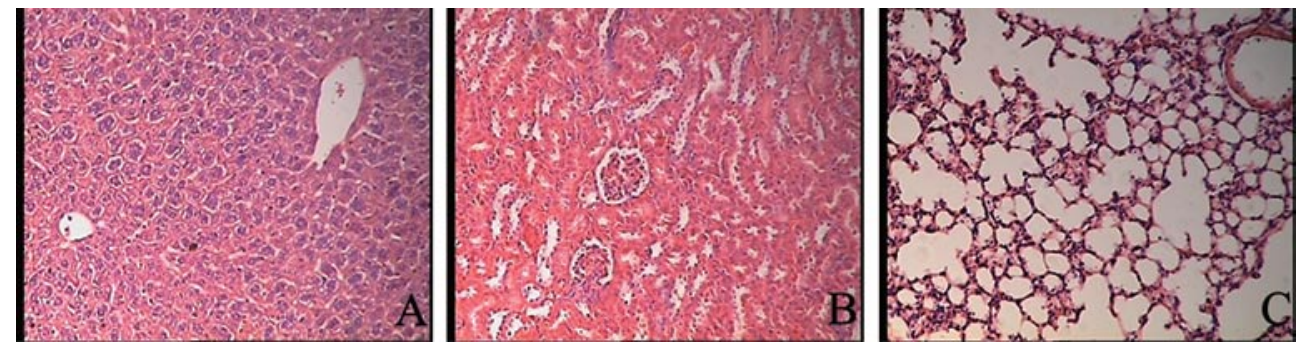

Figure 8. The safety and adverse effects of the Ad-LRIG1 intratumoral injections on athymic mice and histology of the liver, kidney and lungs. Photomicrographs, under light microscopy, of (A) liver, (B) kidney and (C) lungs from Ad-LRIGl-administered athymic mice were captured at the conclusion of the study (H\&E, x200). 
ambiguous, as it was found to be overexpressed in some malignant lesions, including prostate cancer and astrocytoma $(9,24)$. Several hypotheses have been considered to explain the above phenomenon. The activities of the receptor tyrosine kinases (RTKs), inhibited by LRIG1, may either be tumorpromoting or tumor-suppressing, and some predominantly tumor-suppressing receptors may be an LRIG1-negative regulation target (9). Thus whether LRIG1 fragments suppress or promote tumor growth in vivo is currently unknown.

To our knowledge, this is the first study using an adenoviral vector to transfer LRIG1 into an aggressive human bladder cancer xenograft model. According to the results of the tumor growth curve and inhibition rate, xenograft volume growth was markedly retarded after final Ad-LRIG1 administration compared to the control groups. These results suggest that LRIG1 plays a tumor-suppressive role in vivo, in bladder cancer.

As discussed previously, LRIG1 suppresses growth factor signaling by multiple mechanisms, and the EGFR pathway may be a critical one. An important observation of our study is that LRIG1 overexpression in the Ad-LRIG1-treated group correlated with decreased expression of EGFR, which corroborated our studies in vitro (7). The mechanisms of the above phenomena may be explained by previous studies showing that LRIG1 combined with EGFR and enhanced the complex ubiquitination and degradation in a cbl E3 ubiquitin ligase-dependent manner (9). Yet Sweeney et al reported dominant-negative cbl was unable to interfere with LRIG1induced basal loss of receptors, indicating that some other ubiquitin ligase may be involved in LRIG1-mediated EGFR degradation (25).

The nuclear antigen PCNA and $\mathrm{Ki}-67$ are related to cell proliferation and are overexpressed in the $S, G_{2}$, and $M$ phases of the cell cycle, but absent in the $\mathrm{G}_{0}$ phase. High PCNA and $\mathrm{Ki}-67$ labeling indices were found to predict a worse prognosis (18). The evidence from our results showed that expression of the proliferation markers, Ki-67 and PCNA, was reduced in the Ad-LRIG1 group, and indicated that LRIG1-suppressed tumor proliferation may be one of the mechanisms involved in tumor growth suppression. These data were consistent with previous reports in vitro which showed that LRIG1 inhibits proliferation and modulates the cell cycle in cancer cell lines.

Tumors receive nutrients by diffusion when the maximum tumor diameters are smaller than $2 \mathrm{~mm}$, while exponential tumor growth depends upon the development of an adequate blood vasculature. Tumors in the pre-vascular phase are usually associated with local, non-metastatic tumors, whereas tumors in the vascular stage proceed to the process of invasion and metastasis (26). Different from normal blood vessels, the basement membrane of microvessels are not integral and are deficient in mural cells, which may promote tumor cell intravasation and initiate invasion and metastasis (27). Compared to studies in vitro, one of the most important features of xenograft animal models is the ability to assess the anti-angiogenetic effect of therapeutic agents. Few studies had been conducted to demonstrate whether LRIG1 is involved in the tumor angiogenesis process. In our study, we found that, upon administration of LRIG1, microvessel density was dramatically reduced in the human tumor xenografts, and cell ischemia and necrosis were evident in areas of deficient blood supply.
Despite the fact that EGFR- and angiogenesis-targeted therapies are traditionally discussed separately, the linkage between them has been recently studied. It was reported that anti-EGFR therapy leads to a reduction in angiogenesis factors, such as VEGF, IL-8, and bFGF (27). But the mechanisms by which EGFR signaling pathways regulate these factors are still unclear. Thus, whether the reduction in microvessel density directly results from LRIG1 modulation or is the consequence of reduced tumor volume is not clear, and warrants further investigation.

Previous studies using an adenoviral vector have demonstrated successful gene transfer, but the effects of the adenoviral vector in vivo are still ambiguous. Miller et al reported that adenovirus infection enhanced dendritic cell immunostimulatory properties and induced NK cell-mediated tumor protection (28). On the contrary, Inoue et al found that the adenovirus alone accelerated tumor growth in an athymic mouse xenograft model (29). In our study, the xenografts treated with DMEM and Ad-GFP showed no significant difference in volume as determined by the growth curve, nor in expression levels of PCNA and Ki-67 ( $>>0.05)$. The results above demonstrated that the adenoviral vector may have little effect on the athymic mouse immune system and tumor cell proliferation. This also implies that differences in tumor growth among control, Ad-GFP and Ad-LRIG1 groups were mainly attributable to LRIG1 upregulation.

In previous studies, use of the adenoviral vector in vivo has been hampered by toxicity and immune responses. But direct delivery of the adenoviral vector into target organs may minimize these adverse effects (30). It is obvious that bladder cancer is an ideal target for gene therapy, since the agents can be delivered directly into the bladder, and provide ribonuclease, tumor-suppressor gene expression with minimal dissemination into the circulatory system. In the present study, by targeting EGFR, the adenoviral vector or LRIG1 did not cause lesions in important organs, B lymphocyte immunity and humoral immunity of mice. However, despite the advantage mentioned above, one limitation of this study was in assessing adenoviral vector effect and safety. $\mathrm{T}$ lymphocyte immunity response could not be evaluated, because of thymus dysplasia, a unique feature of athymic mice. Therefore, further study of the effects of the adenoviral vector and LRIG1 on non-athymic animals needs to be conducted.

In conclusion, our results contribute to a better realization that strategies based on the downregulation of EGFR expression by LRIG1 may comprise a novel therapeutic approach for improving the prognosis of invasive bladder cancer.

\section{Acknowledgements}

This study was supported by Chinese National Nature and Science fund (nos. 30500521 and 30872653). We are grateful to Professor Håkan Hedman and Dr Jonas Nilsson for providing the pLRIG1-GFP plasmid, and Dr Zhulin Liang for providing the cell line. Wei Guan, Baofeng Wang and Mulan Yang are acknowledged for their kind assistance with the technical support.

\section{References}

1. Dinney CP, McConkey DJ, Millikan RE, et al: Focus on bladder cancer. Cancer Cell 6: 111-116, 2004. 
2. Said N and Theodorescu D: Pathways of metastasis suppression in bladder cancer. Cancer Metastasis Rev 28: 327-333, 2009.

3. Villares GJ, Zigler M, Blehm K, Bogdan C, McConkey D, Colin D and Bar-Eli M: Targeting EGFR in bladder cancer. World J Urol 25: 573-579, 2007.

4. Neal DE and Mellon K: Epidermal growth factor receptor and bladder cancer: a review. Urol Int 48: 365-371, 1992.

5. Kassouf W, Black PC, Tuziak T, et al: Distinctive expression pattern of ErbB family receptors signifies an aggressive variant of bladder cancer. J Urol 179: 353-358, 2008.

6. Bhuvaneswari R, Gan YY, Soo KC and Olivo M: Targeting EGFR with photodynamic therapy in combination with Erbitux enhances in vivo bladder tumor response. Mol Cancer 8: 94, 2009.

7. Yang WM, Yan ZJ, Ye ZQ and Guo DS: LRIG1, a candidate tumour-suppressor gene in human bladder cancer cell line BIU87. BJU Int 98: 898-902, 2006.

8. Nilsson J, Vallbo C, Guo D, Golovleva I, Hallberg B, Henriksson R and Hedman $\mathrm{H}$ : Cloning, characterization, and expression of human LIG1. Biochem Biophys Res Commun 284: 1155-1161, 2001.

9. Hedman $\mathrm{H}$ and Henriksson R: LRIG inhibitors of growth factor signalling-double-edged swords in human cancer? Eur J Cancer 43: 676-682, 2007.

10. Gur G, Rubin C, Katz M, et al: LRIG1 restricts growth factor signaling by enhancing receptor ubiquitylation and degradation. EMBO J 23: 3270-3281, 2004.

11. Slos P, De Meyer M, Leroy P, Rousseau C and Acres B: Immunotherapy of established tumors in mice by intratumoral injection of an adenovirus vector harboring the human IL-2 cDNA: induction of CD8(+) T-cell immunity and NK activity. Cancer Gene Ther 8: 321-332, 2001.

12. Sano D, Kawakami M, Fujita K, et al: Antitumor effects of ZD6474 on head and neck squamous cell carcinoma. Oncol Rep 17: 289-295, 2007

13. Teranishi N, Naito Z, Ishiwata T, et al: Identification of neovasculature using nestin in colorectal cancer. Int J Oncol 30: 593-603, 2007.

14. Tanemura A, Nagasawa T, Inui S and Itami S: LRIG-1 provides a novel prognostic predictor in squamous cell carcinoma of the skin: immunohistochemical analysis for 38 cases. Dermatol Surg 31: 423-430, 2005

15. Axelsson H, Lönnroth $\mathrm{C}$, Andersson $\mathrm{M}$ and Lundholm $\mathrm{K}$ : Mechanisms behind COX-1 and COX-2 inhibition of tumor growth in vivo. Int J Oncol 37: 1143-1152, 2010.

16. Goldstein NS and Armin M: Epidermal growth factor receptor immunohistochemical reactivity in patients with American Join Committee on Cancer Stage IV colon adenocarcinoma: implications for a standardized scoring system. Cancer 92: 1331-1346, 2001.
17. Bibeau F, Boissière-Michot F, Sabourin JC, et al: Assessment of epidermal growth factor receptor (EGFR) expression in primary colorectal carcinomas and their related metastases on tissue sections and tissue microarray. Virchows Arch 449: 281-287, 2006.

18. Korkolopoulou P, Christodoulou P, Konstantinidou AE, ThomasTsagli E, Kapralos P and Davaris P: Cell cycle regulators in bladder cancer: a multivariate survival study with emphasis on p27Kip1. Hum Pathol 31: 751-760, 2000.

19. Weidner N, Semple JP, Welch WR and Folkman J: Tumor angiogenesis and metastasis-correlation in invasive breast carcinoma. N Engl J Med 324: 1-8, 1991.

20. Ma YP, Yang Y, Zhang S, et al: Efficient inhibition of lung cancer in murine model by plasmid-encoding VEGF short hairpin RNA in combination with low-dose DDP. J Exp Clin Cancer Res 29: 56, 2010.

21. Zhao Y, Li Y, Liu J, et al: Adenoviral-vector mediated transfer of HBV-targeted ribonuclease can inhibit HBV replication in vivo. Biochem Biophys Res Commun 371: 541-545, 2008.

22. St. George JA: Gene therapy progress and prospects: adenoviral vectors. Gene Ther 10: 1135-1141, 2003.

23. Thomasson M, Hedman H, Guo D, Ljungberg B and Henriksson R: LRIG1 and epidermal growth factor receptor in renal cell carcinoma: a quantitative RT-PCR and immunohistochemical analysis. Br J Cancer 89: 1285-1289, 2003.

24. Lapointe J, Li C, Higgins JP, et al: Gene expression profiling identifies clinically relevant subtypes of prostate cancer. Proc Natl Acad Sci USA 101: 811-816, 2004.

25. Sweeney C, Miller JK, Shattuck DL and Carraway KL: ErbB receptor negative regulatory mechanisms: implications in cancer. J Mammary Gland Biol Neoplasia 11: 89-99, 2006.

26. Folkman J, Watson K, Ingber D and Hanahan D: Induction of angiogenesis during the transition from hyperplasia to neoplasia. Nature 339: 58-61, 1989.

27. Bellmunt J, Hussain M and Dinney CP: Novel approaches with targeted therapies in bladder cancer. Therapy of bladder cancer by blockade of the epidermal growth factor receptor family. Crit Rev Oncol Hematol 46: S85-S104, 2003.

28. Miller G, Lahrs S, Pillarisetty VG, Shah AB and DeMatteo RP: Adenovirus infection enhances dendritic cell immunostimulatory properties and induces natural killer and T-cell-mediated tumor protection. Cancer Res 62: 5260-5266, 2002.

29. Inoue K, Perrotte P, Wood CG, Slaton JW, Sweeney P and Dinney CP: Gene therapy of human bladder cancer with adenovirus-mediated antisense basic fibroblast growth factor Clin Cancer Res 6: 4422-4431, 2000.

30. Malmström PU, Loskog AS, Lindqvist CA, et al: AdCD40L immunogene therapy for bladder carcinoma - the first phase I/IIa trial. Clin Cancer Res 16: 3279-3287, 2010. 